\title{
The Turbulent Shock Origin of Proto-Stellar Cores
}

\section{Citation}

Padoan, Paolo, Mika Juvela, Alyssa A. Goodman, and Ake Nordlund. 2001. “The Turbulent Shock Origin of Proto-Stellar Cores." The Astrophysical Journal 553 (1): 227-34. https:// doi.org/10.1086/320636.

\section{Permanent link}

http://nrs.harvard.edu/urn-3:HUL.InstRepos:41397491

\section{Terms of Use}

This article was downloaded from Harvard University's DASH repository, and is made available under the terms and conditions applicable to Other Posted Material, as set forth at http:// nrs.harvard.edu/urn-3:HUL.InstRepos:dash.current.terms-of-use\#LAA

\section{Share Your Story}

The Harvard community has made this article openly available.

Please share how this access benefits you. Submit a story.

\section{Accessibility}




\title{
THE TURBULENT SHOCK ORIGIN OF PROTO-STELLAR CORES
}

\author{
Paolo Padoan ${ }^{1}$ \\ Harvard-Smithsonian Center for Astrophysics, 60 Garden Street, Cambridge, MA 02138 \\ MiKa JuVELA ${ }^{2}$ \\ Helsinki University Observatory, Tähtitorninmäki, PO Box 14, SF-00014 University of Helsinki, Finland \\ AlysSA A. GoODMAN ${ }^{3}$ \\ Harvard-Smithsonian Center for Astrophysics, 60 Garden Street, Cambridge, MA 02138 \\ AND \\ ÅKE NORDLUND ${ }^{4}$ \\ Astronomical Observatory and Theoretical Astrophysics Center, Juliane Maries Vej 30, DK-2100 Copenhagen, Denmark \\ Received 2000 July 30; accepted 2001 January 16
}

\begin{abstract}
The fragmentation of molecular clouds (MC) into proto-stellar cores is a central aspect of the process of star formation. Because of the turbulent nature of supersonic motions in MCs, it has been suggested that dense structures such as filaments and clumps are formed by shocks in a turbulent flow. In this work we present strong evidence in favor of the turbulent origin of the fragmentation of MCs. The most generic result of turbulent fragmentation is that dense postshock gas traces a gas component with a smaller velocity dispersion than lower density gas, since shocks correspond to regions of converging flows, where the kinetic energy of the turbulent motion is dissipated. Using synthetic maps of spectra of molecular transitions, computed from the results of numerical simulations of supersonic turbulence, we show that the dependence of velocity dispersion on gas density generates an observable relation between the rms velocity centroid and the integrated intensity (column density), $\sigma\left(V_{0}\right)-I$, which is indeed found in the observational data. The comparison between the theoretical model (maps of synthetic ${ }^{13} \mathrm{CO}$ spectra) with ${ }^{13} \mathrm{CO}$ maps from the Perseus, Rosette, and Taurus MC complexes shows excellent agreement in the $\sigma\left(V_{0}\right)-I$ relation. The $\sigma\left(V_{0}\right)-I$ relation of different observational maps with the same total rms velocity are remarkably similar, which is a strong indication of their origin from a very general property of the fluid equations, such as the turbulent fragmentation process.

Subject headings: ISM: individual (Perseus, Rosette, Taurus) - ISM: kinematics and dynamics radio lines: ISM - turbulence

On-line material: color figures
\end{abstract}

\section{INTRODUCTION}

The importance of turbulence in the process of star formation was recognized long ago (Von Weizsäcker 1951), and was discussed in a seminal paper by Larson (1981). Subsequent works have tried to use the observational data to relate different properties of molecular clouds (MCs) with the physics of laboratory and numerical turbulence, such as power spectra of kinetic energy (Leung, Kutner, \& Mead 1982; Myers 1983; Quiroga 1983; Sanders, Scoville, \& Solomon 1985; Dame et al. 1986; Falgarone \& Pérault 1987; Falgarone, Puget, \& Pérault 1992), probability distributions of velocity and velocity differences (Scalo 1984; Kleiner \& Dickman 1985, 1987; Hobson 1992; Miesch \& Bally 1994; Miesch \& Scalo 1995; Lis et al. 1996; Miesch, Scalo, \& Bally 1999), intermittency (Falgarone \& Phillips 1990; Falgarone \& Puget 1995; Falgarone, Pineau Des Forets, \& Roueff 1995), and self-similarity (Beech 1987; Bazell \& Désert 1988; Scalo 1990; Dickman, Margulis, \& Horvath 1990; Falgarone, Phillips, \& Walker 1991; Zimmermann, Stutzki, \& Winnewisser 1992; Henriksen 1991; Hetem \& Lepine 1993; Vogelaar \& Wakker 1994; Elmegreen \& Falgarone 1996).

\footnotetext{
${ }^{1}$ ppadoan@cfa.harvard.edu.

2 juvela@astro.helsinki.fi.

3 agoodman@cfa.harvard.edu.

${ }^{4}$ aake@astro.ku.dk.
}

During the last decade, numerical simulations of transonic turbulence (Passot \& Pouquet 1987; Passot, Pouquet, \& Woodward 1988; Léorat, Passot, \& Pouquet 1990; Lee, Lele, \& Moin 1991; Porter, Pouquet, \& Woodward 1992, 1994; Kimura \& Tosa 1993; Vazquez-Semadeni 1994; Passot \& Vazquez-Semadeni 1995) and highly supersonic turbulence (Passot \& Vázquez-Semadeni 1995; VázquezSemadeni, Passot, \& Pouquet 1996; Padoan \& Nordlund 1997, 1999; Stone, Ostriker, \& Gammie 1998; Mac Low et al. 1999; Ostriker, Gammie, \& Stone 1999; Padoan, Zweibel, \& Nordlund 2000c; Klessen, Heitsch, \& Mac Low 2000) on relatively high resolution numerical grids have become available, and very detailed comparisons between observational data and turbulence models of MCs have been performed (Padoan, Jones, \& Nordlund 1997; Padoan et al. 1998, 1999; Padoan \& Nordlund 1999; Rosolowsky et al. 1999; Padoan, Rosolowsky, \& Goodman 2000b).

Recent numerical studies of supersonic magnetohydrodynamic (MHD) turbulence have brought a new understanding of the physics of turbulence. The most important results are:

1. Supersonic turbulence decays in approximately one dynamical time, independent of the magnetic field strength (Padoan \& Nordlund 1997, 1999; Mac Low et al. 1999; Stone et al. 1998; MacLow 1999).

2. The probability distribution of gas density in isother- 
mal turbulence is well approximated by a lognormal distribution, whose standard deviation is a function of the rms Mach number of the flow (Vazquez-Semadeni 1994; Padoan 1995; Padoan et al. 1997; Scalo et al. 1998; Nordlund \& Padoan 1999; Ostriker et al. 1999).

3. Supersonic isothermal turbulence generates a complex system of shocks, which fragment the gas very efficiently into high-density sheets, filaments, and cores (this is the general result of any numerical simulation of supersonic turbulence).

4. Super-Alfvénic turbulence provides a good description of the dynamics of MCs, and an explanation for the origin of dense cores with magnetic field strength consistent with Zeeman splitting observations. (Padoan \& Nordlund 1997, 1999). ${ }^{5}$

We call "turbulent fragmentation" the process of generating high-density structures by turbulent shocks. Since random supersonic motions are ubiquitous in MCs, turbulent fragmentation cannot be avoided: it is a direct consequence of the observational evidence. In some analytical studies it is tacitly assumed that turbulent fragmentation can be neglected if the kinetic energy of random motions is in rough equipartition with the magnetic energy. This assumption is wrong, because motions along magnetic field lines are unavoidable, and so turbulent fragmentation occurs via supersonic compressions along the magnetic field lines, as discussed by Gammie \& Ostriker (1996) and Padoan \& Nordlund $(1997,1999)$.

Numerical simulations of turbulence have been used to discuss the turbulent origin of MC structures by Passot \& Pouquet (1987). Vazquez-Semadeni et al. (1996) and Ballesteros-Paredes, Hartmann, \& Vazquez-Semadeni (1999) have used two-dimensional simulations to argue that MCs are formed by turbulence. Padoan \& Nordlund (1997, 1999) have shown that supersonic and super-Alfvénic turbulence can explain the origin of magnetized cores in MCs, including the observed field strength-density $(B-n)$ relation (Myers \& Goodman 1988; Fiebig \& Güsten 1989; Crutcher 1999). The idea that proto-stellar cores and stars are formed in turbulent shocks has been previously discussed by Elmegreen (1993). An analysis of the gravitational instability of the cores can be found in that work. Here we present new strong observational evidence in favor of the turbulent shock origin of proto-stellar cores. Such evidence is based on the fact that dense postshock gas traces a gas component with a smaller velocity dispersion than lower density gas, since it maps regions of converging flows, where the kinetic energy of the turbulent motion is dissipated.

In $\S \S 2$ and 3, the numerical simulations and observational data used in this work are briefly described. In $\S 4$ we compute the rms flow velocity as a function of the gas density in simulations of supersonic turbulence, and show that it decreases for increasing values of the gas density. In $\S 5$ we show that such general property generates an observable relation between the rms velocity centroid and the integrated intensity (roughly proportional to the surface density) for the $J=1-0{ }^{13} \mathrm{CO}$ transition, and in $\S 6$ the same relation is found in the observational data. Results are discussed and conclusions are summarized in $\S 7$.

\footnotetext{
${ }^{5}$ Other groups have argued that sub- or trans-Alfvénic turbulence provides as good a match to the observed properties of self-gravitating magnetized molecular clouds (Ostriker et al. 1999, 2001).
}

\section{NUMERICAL MODELS}

The numerical models used in this work are based on the results of numerical simulations of super-Alfvénic and highly supersonic MHD turbulence, run on a $128^{3}$ computational mesh, with periodic boundary conditions. As in our previous works, the initial density and magnetic fields are uniform; the initial velocity is random, generated in Fourier space with power only on the large scale. We also apply an external random force, to drive the turbulence at a roughly constant rms Mach number of the flow. This force is generated in Fourier space, with power only on small wavenumbers $(1<k<2)$, as the initial velocity. The isothermal equation of state is used. Descriptions of the numerical code used to solve the MHD equations can be found in Galsgaard \& Nordlund (1996), Nordlund, Stein, \& Galsgaard (1996), Nordlund \& Galsgaard (1997), and Padoan \& Nordlund (1999).

In this work we neglect the effect of self-gravity, although that can be described with a different version of our code (Padoan et al. 2000a). Here we compare the relative velocity of regions of $\mathrm{MC}$ complexes as a function of their gas density or column density. Such regions are distributed across the full extension of the MC complexes, which is several pc. In our numerical models with self-gravity, driven continuously by an external force on the large scale, selfgravity is responsible for the collapse of gravitationally bound cores formed by the turbulent flow, but does not significantly affect the large-scale flow. Since we assume that large-scale motions in MC complexes are due to turbulence, and not to a gravitational collapse, we can neglect selfgravity. Similarly, we have neglected the effect of ambipolar drift, since it is not relevant for motions on the scale of several pc, although that is computed in a different version of our code, using the strong-coupling approximation (see Padoan et al. 2000c).

In order to scale the models to physical units, we use the following empirical Larson-type relations, as in our previous works:

$$
\mathscr{M}=2.0\left(\frac{L}{1 \mathrm{pc}}\right)^{0.5}
$$

where $\mathscr{M}$ is the rms sonic Mach number of the flow, a temperature of $T=10 \mathrm{~K}$ is assumed, and

$$
\langle n\rangle=2.0 \times 10^{3}\left(\frac{L}{1 \mathrm{pc}}\right)^{-1},
$$

where the gas density $n$ is expressed in $\mathrm{cm}^{-3}$. The rms sonic Mach number is an input parameter of the numerical simulations, and can be used to rescale them to physical units. When comparing theoretical models with observations, $\mathscr{M}$ is in fact the only parameter that we need to match (or its two-dimensional equivalent on the maps, $\sigma_{v}$; see $\S 3$ ). In the absence of self-gravity and magnetic field, the statistical properties of turbulent flows (very large Reynolds number) with the same value of $\mathscr{M}$ should be universal, and independent of the average density (for isothermal flows without self-gravity). If the magnetic field is present, the rms Alfvénic Mach number of the flow is also an input parameter of the numerical simulations (it determines the magnetic field strength). The physical unit of velocity in the code is the isothermal speed of sound, $C_{s}$, and the physical unit of the magnetic field is $C_{s}(4 \pi\langle\rho\rangle)^{1 / 2}$ (cgs). 
TABLE 1

PARAMETERS OF ObSERVATIONAL MAPS

\begin{tabular}{lccccccc}
\hline \hline Cloud Name & $\begin{array}{c}L \\
(\mathrm{pc})\end{array}$ & $\begin{array}{c}\sigma_{v} \\
\left(\mathrm{~m} \mathrm{~s}^{-1}\right)\end{array}$ & $\begin{array}{c}d x \\
(\mathrm{pc})\end{array}$ & $\begin{array}{c}d v \\
\left(\mathrm{~km} \mathrm{~s}^{-1}\right)\end{array}$ & $\begin{array}{c}N \\
(\mathrm{~K})\end{array}$ & $\langle Q\rangle$ & Reference \\
\hline Taurus ...... & 38 & 1.0 & 0.10 & 0.10 & 0.24 & 2.3 & 1 \\
Perseus ...... & 27 & 2.0 & 0.15 & 0.27 & 0.24 & 2.8 & 2 \\
Rosette ...... & 52 & 2.4 & 0.84 & 0.68 & 0.20 & 2.1 & 3 \\
& 36 & 2.2 & 0.84 & 0.68 & 0.19 & 4.0 & 4 \\
& 36 & 2.0 & 0.42 & 0.06 & 0.12 & 3.8 & 5
\end{tabular}

NoTE.-Col. (2): maximum spatial extension; col. (3) total rms velocity; col. (4) telescope beam size; col. (5) velocity channel width; col. (6) rms noise; col. (7) average spectrum quality $(\mathrm{S} / \mathrm{N})$.

REFERENCES.- (1) Mizuno et al. 1995; (2) Y. Billawala et al., in preparation; (3) Blitz \& Stark 1986; (4) Blitz \& Stark (matching region); (5) Heyer et al., in preparation.

In this work we use two numerical models with rms velocity 3.4 and $1.7 \mathrm{~km} \mathrm{~s}^{-1}$, which correspond to $\mathscr{M} \approx 13.0$ and 6.5 , respectively. Using the Larson-type relations given in equations (1) and (2), we get $L \approx 42$ and $10.5 \mathrm{pc}$ and $\langle n\rangle \approx 48$ and $190 \mathrm{~cm}^{-3}$, respectively. The one-dimensional rms velocities for the two models are $\sigma_{v}=2.0$ and $1.0 \mathrm{~km}$ $\mathrm{s}^{-1}$, which are also recovered from the analysis of the synthetic spectral maps computed with these models. The values of $\sigma_{v}$ have been chosen for the appropriate comparison with the observational data presented in $\S 3 .^{6}$ The magnetic field strength is $B \approx 5 \mu \mathrm{G}$ in both models.

Maps of synthetic spectra of molecular transitions are computed using a non-LTE Monte Carlo radiative transfer code (Juvela 1997, 1998) from the three-dimensional density and velocity fields generated in the numerical MHD experiments. The method of computing synthetic spectra was presented in Padoan et al. (1998). For the purpose of this work, we use only one molecular transition, namely, $J=1-0$ ${ }^{13} \mathrm{CO}$. A uniform temperature of $T=10 \mathrm{~K}$ is assumed for these radiative transfer calculations, in agreement with the isothermal equation of state used in the MHD calculations. We are currently computing thermal equilibrium models of MCs, which we will use in a future work to study the effect of realistic temperature variations in molecular spectra.

Since the spectral noise resulting from uncertainties in our radiative transfer calculations is always much smaller than the typical observational noise, the comparison of synthetic spectra with observational data can be done only after adding noise to the synthetic spectra, and the effect of noise on the statistical properties of the spectra needs to be quantified (see $\S 4$ ).

\section{OBSERVATIONAL DATA}

We choose to use the $J=1-0{ }^{13} \mathrm{CO}$ transition because it samples the range of values of column density we are interested in here, and also because several large maps of molecular clouds are available in this transition. We compare maps of $J=1-0{ }^{13} \mathrm{CO}$ synthetic spectra with some of the largest observational $J=1-0{ }^{13} \mathrm{CO}$ spectral maps in the literature from the following Galactic regions: the Perseus MC complex (Y. Billawala et al., in preparation), the Taurus MC complex (Mizuno et al. 1995), and the Rosette MC complex (Blitz \& Stark 1986; Heyer et al., in preparation).

\footnotetext{
${ }^{6}$ The Taurus MC complex has $\sigma_{v} \approx 1.0 \mathrm{~km} \mathrm{~s}^{-1}$ and $L \approx 40 \mathrm{pc}$, while the Larson-type relation of equation (1) would give $L \approx 12.6 \mathrm{pc}$. For the purpose of this work, we are interested in comparing the observations with numerical models with similar rms Mach number, and we do not try to match the physical extension of each MC complex.
}

These MC complexes have an extension of approximately $30-50 \mathrm{pc}$, and radial velocity dispersions in the range 1-2.4 $\mathrm{km} \mathrm{s}^{-1}$. We define the total rms velocity of a map as the rms velocity weighted with the total spectrum of the map, $T(v)$ :

$$
\sigma_{v}=\sqrt{\sum_{v} \frac{(v-\bar{v})^{2} T(v) d v}{\sum_{v} T(v) d v}}
$$

where

$$
\bar{v}=\frac{\sum_{v} v T(v) d v}{\sum_{v} T(v) d v} .
$$

The Blitz \& Stark map of the Rosette MC complex has $\sigma_{v}=2.4 \mathrm{~km} \mathrm{~s}^{-1}$, but if this is limited to the region that matches the more recent Heyer et al. map the value is $\sigma_{v}=$ $2.0 \mathrm{~km} \mathrm{~s}^{-1}$ (for both maps). The full map of the Perseus MC complex also yields a value of $\sigma_{v}=2.0 \mathrm{~km} \mathrm{~s}^{-1}$. Taurus is instead much less turbulent, despite its large spatial extent, with $\sigma_{v}=1.0 \mathrm{~km} \mathrm{~s}^{-1}$.

The angular resolution is inversely proportional to the diameter of the antenna: $4 \mathrm{~m}$ for the Taurus map, $7 \mathrm{~m}$ for the Perseus and the Blitz \& Stark (1986) Rosette maps, and $14 \mathrm{~m}$ for the Heyer et al. (in preparation) map of Rosette. Assuming a distance of 140 pc for Taurus, 300 pc for Perseus, and $1600 \mathrm{pc}$ for Rosette, the spatial resolution of the maps is $0.1 \mathrm{pc}$ for Taurus, $0.15 \mathrm{pc}$ for Perseus, $0.84 \mathrm{pc}$ for the Blitz \& Stark map of Rosette, and 0.42 pc for the Heyer et al. map of Rosette. The spectral resolution is $0.1 \mathrm{~km} \mathrm{~s}^{-1}$ for Taurus, $0.273 \mathrm{~km} \mathrm{~s}^{-1}$ for Perseus, $0.68 \mathrm{~km} \mathrm{~s}^{-1}$ for the Blitz \& Stark map of Rosette, and $0.06 \mathrm{~km} \mathrm{~s}^{-1}$ for the Heyer et al. map of the same cloud.

The rms noise $N$ and average spectrum quality $\langle Q\rangle$ (see Padoan et al. 2000b) also vary from map to map. The spectrum quality, $Q$, is related to the signal-to-noise ratio, $\mathrm{S} / \mathrm{N}$. It is defined as the ratio of the rms signal (over the whole spectrum or inside a velocity window) to the rms noise, $N$ :

$$
Q=\frac{\sqrt{\sum_{v} T(v)^{2} d v}}{N}
$$

The usual definition of $\mathrm{S} / \mathrm{N}$ is based on Gaussian fits of the spectra, which we prefer to avoid because the $J=1-0{ }^{13} \mathrm{CO}$ transition typically yields spectra with significant nonGaussian shapes and multiple components. The spectrum quality is a sort of $\mathrm{S} / \mathrm{N}$ weighted over the whole spectrum. The relation between $Q$ and $\mathrm{S} / \mathrm{N}$ is discussed in Padoan et al. (2000b). We define the average spectrum quality $\langle Q\rangle$ as the value of $Q$ averaged over the whole map. Values of $\langle Q\rangle$, $N$, resolutions, and $\sigma_{v}$ are listed in Table 1 for all the obser- 
vational maps. In the following discussion, when different maps are compared with each other, we make noise and velocity resolution equal in the different maps by adding noise and reducing the velocity resolution where necessary.

\section{VELOCITY DISPERSION VERSUS GAS DENSITY IN SUPERSONIC TURBULENCE}

Turbulent fragmentation generates a complex system of dense postshock sheets, filaments, and cores, reminiscent of structures observed in MC maps. An example of a twodimensional projection of the three-dimensional density field from a simulation of supersonic turbulence is shown in Figure 1. In previous works (Padoan et al. 1997; Padoan \& Nordlund 1997, 1999) we have shown that, in addition to this morphological similarity, the density field of numerical supersonic turbulence has important statistical properties in agreement with the density field of observed MCs.

Figure 2 (left) is a two-dimensional section (no projection) of the same three-dimensional density field used in Figure 1. A complex system of filaments is apparent, with a number of high-density cores inside the filaments. In threedimensional supersonic turbulence, filaments are formed by two-dimensional compressions (at the intersection of sheets), and the densest cores are formed by threedimensional compressions. Most of the "filaments" in twodimensional sections, such as in Figure 2, are instead two-dimensional cuts through sheets, and most of the cores are local density maxima due to fluctuations in the shock velocity (they usually corresponds to strongly curved segments of filaments). Such density maxima are often unstable to gravitational collapse, and are the origin of proto-stellar cores.

Since the dense gas originates in shocks (that is, in regions of converging flows), it should move with significantly lower velocity than the lower density turbulent flow. This is illus-

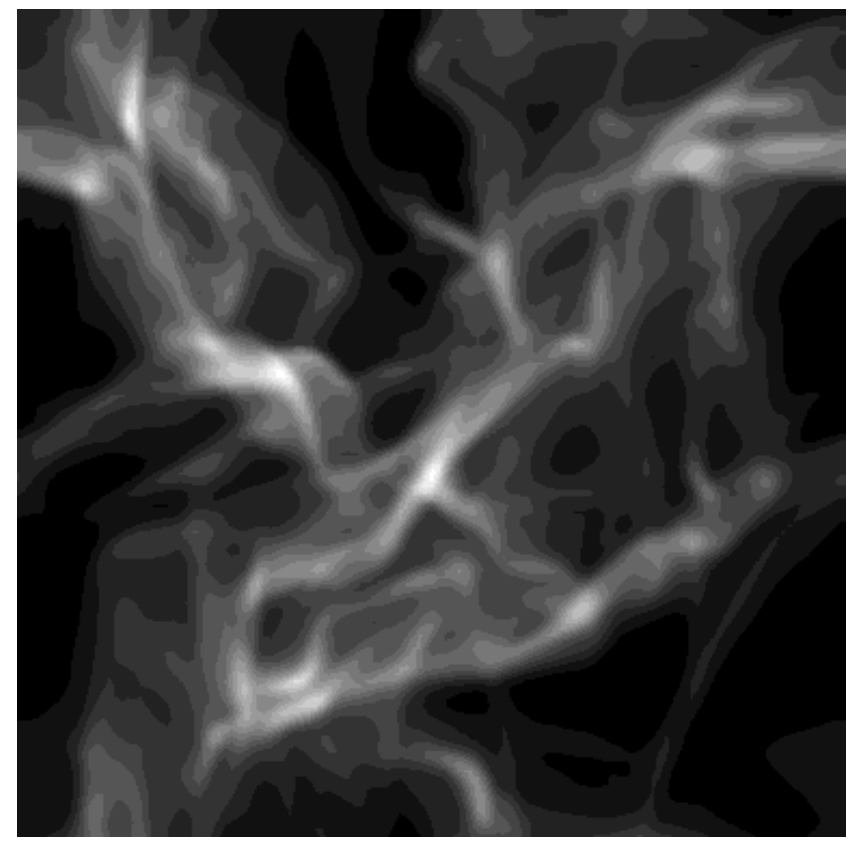

FIG. 1.-Two-dimensional projection of the three-dimensional density field from a simulation of isothermal supersonic turbulence with rms Mach number $\sim 10$. [See the electronic edition of the Journal for a color version of this figure.]

trated in the right panel of Figure 2, which shows the magnitude of the flow velocity on the same plane as the left panel. Dark blue shows low velocity, and dark red high velocity. It is clear that high-density filaments trace regions of low velocity, at the intersections of high-velocity "blobs." This general property of supersonic turbulence is quantified by the dependence of the rms flow velocity, $\sigma(v)$, on the gas

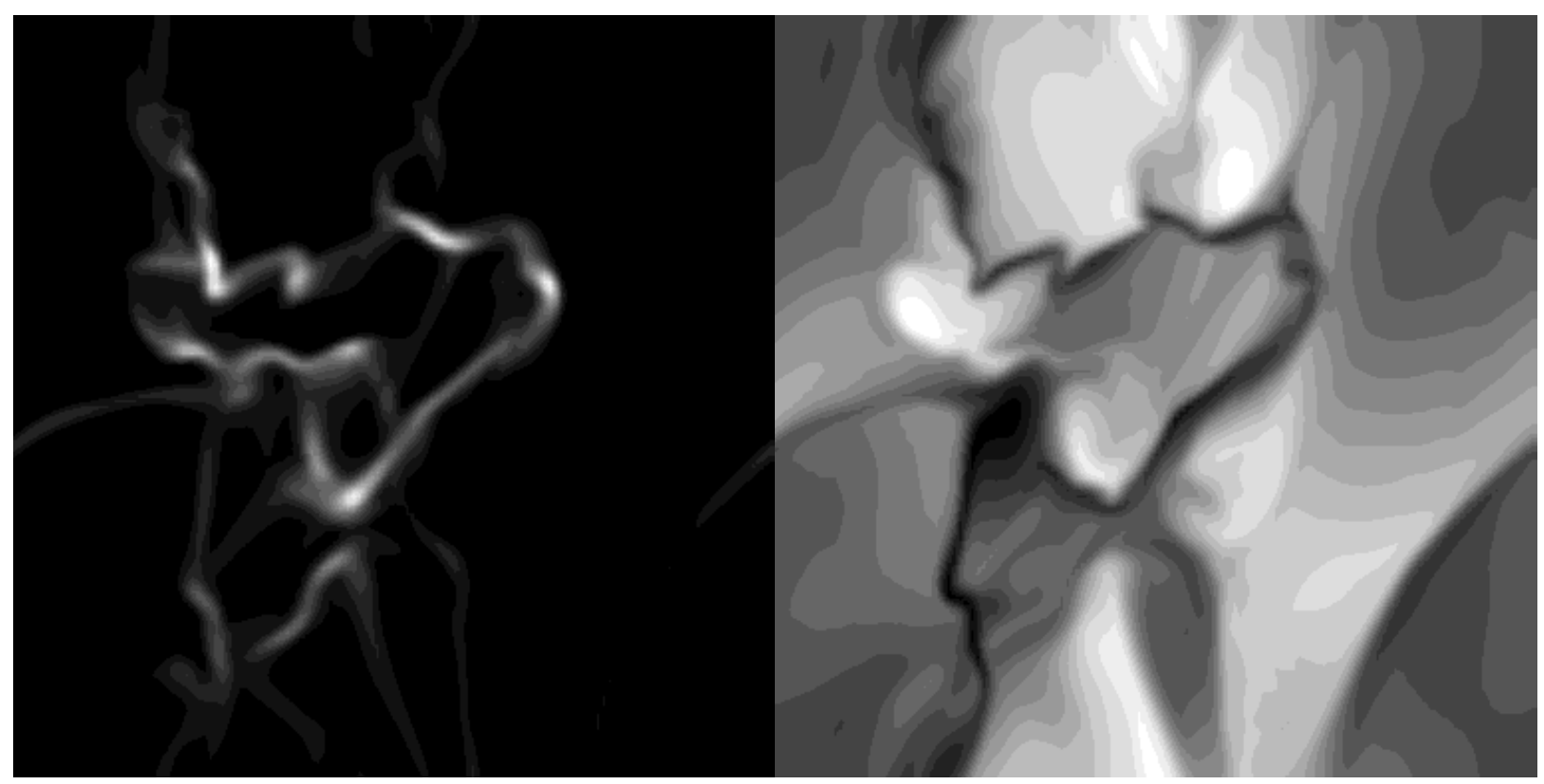

Fig. 2.-Left: Two-dimensional section (no projection) of the same density field used for Fig. 1. Most filaments are sections of sheets, and most dense cores are density maxima inside curved segments of filaments, formed by fluctuations in the shock velocity. Right: Modulus of the three-dimensional flow velocity on the same two-dimensional plane as in the left panel. Dark blue shows low velocity, and dark red high velocity. The dense filaments on the left panel are commonly found in regions of small flow velocity, at the intersections of patches of high velocity. [See the electronic edition of the Journal for a color version of this figure.] 


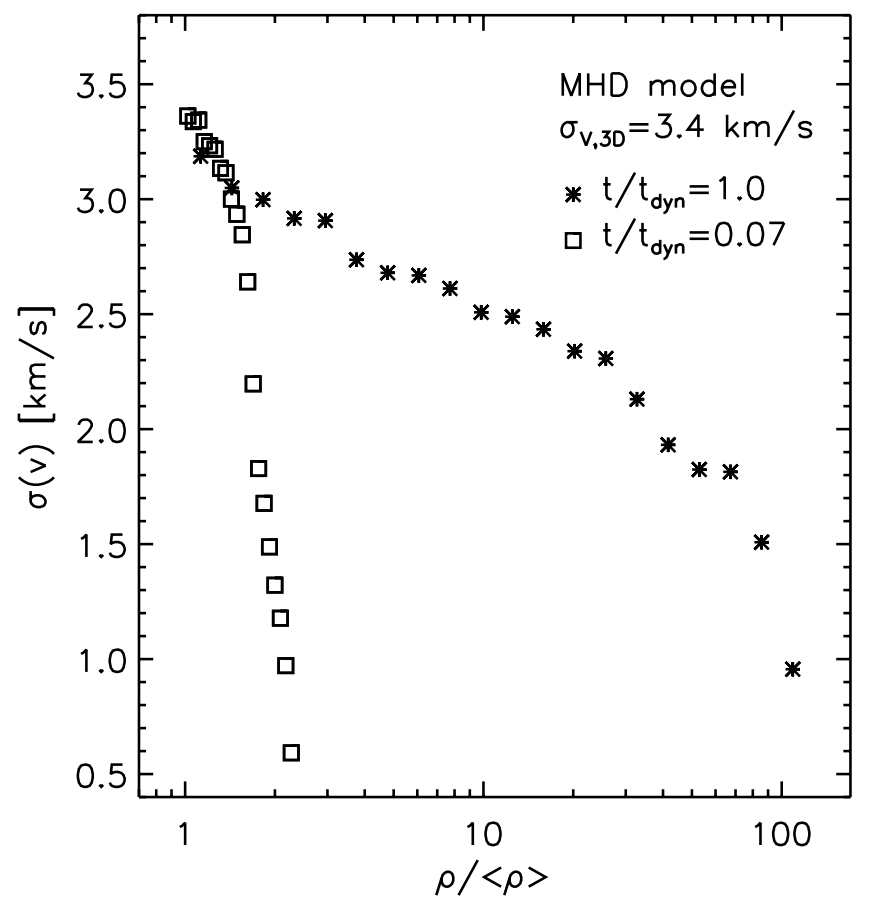

FIG. 3.-Plot of the rms flow velocity, conditioned to gas density, vs. the gas density, computed from a simulation of supersonic turbulence with rms Mach number $\sim 10$. Squares are for an early time, just $7 \%$ of the dynamical time after an initial condition with uniform density; asterisks are for a time equal to a dynamical time. Density values are binned over 20 logarithmic intervals between the average and the highest density. Within each interval, the density grows by a factor of 1.04 at the early time, and 1.27 at one dynamical time.

density,

$$
\sigma(v)=\left\langle(v-\bar{v})^{2} \mid \rho\right\rangle .
$$

We repeat for different values of $\rho$, and then plot the result versus the gas density. Equation (6) means that the rms flow velocity is obtained as an average over the whole computational box, using only positions where the gas density has a value contained in an interval centered around $\rho$. The average is repeated for different intervals of values of $\rho$, to span the whole range of densities.

The plot is shown in Figure 3, at time $t / t_{\mathrm{dyn}}=0.07$ (squares) and $t / t_{\mathrm{dyn}}=1.0$ (asterisks), where $t / t_{\mathrm{dyn}}$ is the time in units of the dynamical time (defined as the size of the computational box divided by the rms flow velocity), and the density is uniform in the initial conditions, at $t / t_{\mathrm{dyn}}=$ 0.0 . At $t / t_{\mathrm{dyn}}=1.0$, regions of $\rho \approx\langle\rho\rangle$ have $\sigma(v)$ comparable to the total $\mathrm{rms}$ velocity, $\sigma_{v}=3.0 \mathrm{~km} \mathrm{~s}^{-1}$, while regions with 10 times higher density have much smaller rms velocity, $\sigma(v) \approx 2.3 \mathrm{~km} \mathrm{~s}^{-1}$, and at $\rho=100\langle\rho\rangle, \sigma(v) \approx 0.9 \mathrm{~km}$ $\mathrm{s}^{-1}$. The rms velocity conditioned to the gas density decreases sharply with increasing gas density already at a very early time, $t / t_{\mathrm{dyn}}=0.07$, when the density field is still very smooth.

\section{RMS VELOCITY CENTROID VERSUS INTEGRATED INTENSITY}

Observational spectral maps of MCs do not provide a direct estimate of the three-dimensional velocity field and gas density. Only radial velocity and velocity-integrated intensity (which is roughly proportional to the surface density), averaged along each line of sight, are directly avail- able from the data. However, the dependence of the rms velocity centroid on the integrated intensity should resemble the $\sigma(v)-\rho$ relation, since lines of sight of high intensity are usually dominated by one or more dense cores. The velocity centroid, $V_{0}$, is the average velocity along an individual line of sight $x$ on the map,

$$
V_{0}(\boldsymbol{x})=\frac{\sum_{v} v T(v, \boldsymbol{x}) d v}{\sum_{v} T(v, \boldsymbol{x}) d v},
$$

where $T(v, x)$ is the signal (antenna temperature) at velocity $v$ and position $\boldsymbol{x}$, and $d v$ is the width of the velocity channel. The integrated intensity, $I(x)$, is

$$
I(\boldsymbol{x})=\sum_{v} T(v, \boldsymbol{x}) d v
$$

We compute the rms velocity centroid, $\sigma\left(V_{0}\right)$, conditioned on $I$,

$$
\sigma\left(V_{0}\right)=\left\langle\left(V_{0}-\bar{V}_{0}\right)^{2} \mid I\right\rangle_{x}
$$

(spatial average), and plot it against $I$. Equation (9) means that the rms velocity centroid is obtained as an average over the whole map, selecting all lines of sight on the map with values of $I$ inside a given interval, $[I, I+d I]$. This rms velocity centroid is not equivalent to a local line width, because it is obtained as an average over the whole map. The plot is shown in Figure 4, for two maps of $J=1-0$ ${ }^{13} \mathrm{CO}$ synthetic spectra with different total line widths $\sigma_{v}$

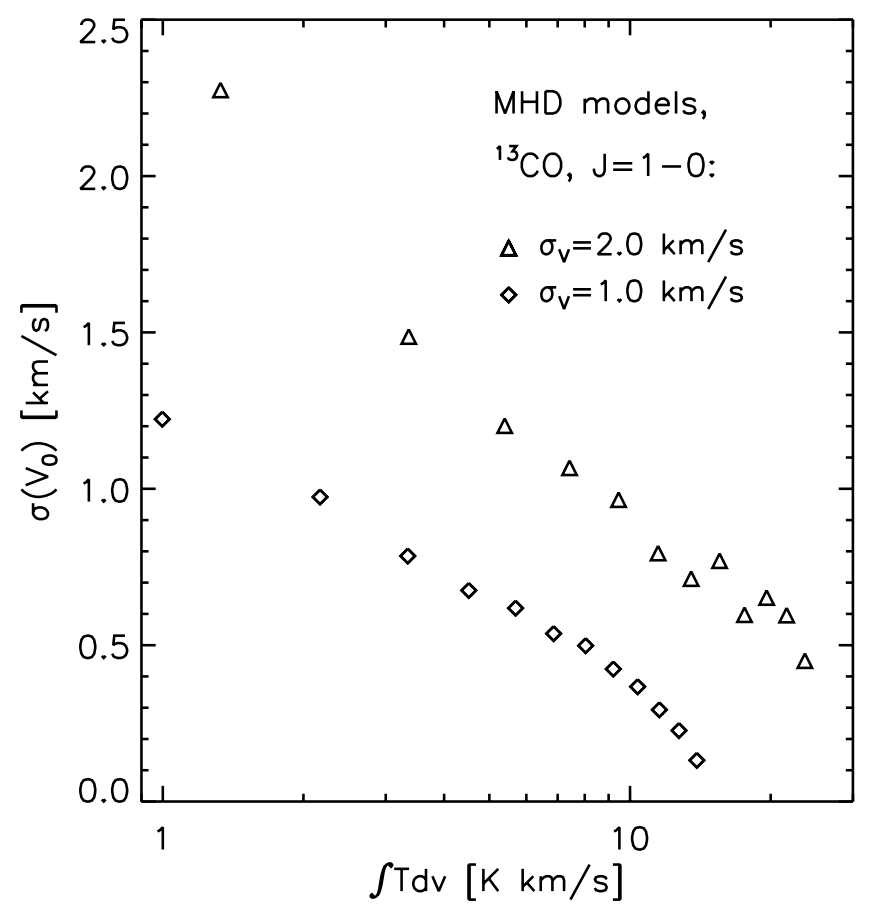

FIG. 4.-Plot of the rms velocity centroid, conditioned to integrated intensity, vs. the integrated intensity (see text for details). Two maps of synthetic spectra from MHD turbulence simulations are used, with different values of the total $\mathrm{rms}$ radial velocity (intensity weighted), $\sigma_{v}=1.0 \mathrm{~km}$ $\mathrm{s}^{-1}$ (diamonds), and $\sigma_{v}=2.0 \mathrm{~km} \mathrm{~s}^{-1}$ (triangles). The model with larger rms velocity also has a slightly larger maximum intensity (surface density), although both models are rescaled to physical units using the Larson relations (see $\S 2$ ). Integrated intensity values are binned over 12 linear intervals between the lowest and the highest values in the map. Within each interval, the density increment is $\approx 1 \mathrm{~K} \mathrm{~km} \mathrm{~s}^{-1}$ (for the $\sigma_{v}=1.0 \mathrm{~km}$ $\mathrm{s}^{-1}$ model) and $\approx 2 \mathrm{~K} \mathrm{~km} \mathrm{~s}^{-1}$ (for the $\sigma_{v}=2.0 \mathrm{~km} \mathrm{~s}^{-1}$ model). 
(defined in $\S 3$ ). Figure 4 shows that $\sigma\left(V_{0}\right)$ decreases significantly for increasing values of $I$. The general property of supersonic turbulence, namely, that high-density gas moves relatively slowly, is therefore also apparent in the observable relation $\sigma\left(V_{0}\right)-I$. The relation $\sigma\left(V_{0}\right)-I$ is affected by noise and by the width of the velocity channels, which must be taken into account when comparing different maps. Noise has been added to the synthetic spectra used for computing the plot shown in Figure 4, to yield a value of the spectrum quality comparable to a typical value found in the observational data used in this work, $\langle Q\rangle=3.5$ (see $\S 3$ for the definition of $\langle Q\rangle$ ).

In Figure 5 we show the effect of noise (left) and spectral resolution (right). The effect of increasing the noise (decreasing the value of $\langle Q\rangle$ ) is to make the $\sigma\left(V_{0}\right)-I$ relation steeper, because the uncertainty in the determination of the velocity centroids due to noise contributes to the dispersion of velocity centroid values, and the effect increases at decreasing values of integrated intensity $I$. Lower spectral resolution further increases the same effect. However, we have verified that if the noise is low enough, $\langle Q\rangle>20$, neither noise nor velocity resolution have an effect on the $\sigma\left(V_{0}\right)-I$ relation, and the squares in the left panel of Figure 5 therefore correspond to the intrinsic relations. We can conclude that this observable relation truly originates from the three-dimensional $\sigma(v)-\rho$ relation, with only a partial contribution from noise.

We have also verified that spatial resolution does not affect our results. The spatial resolution can be decreased significantly, by rebinning the map to a smaller number of spectra, without any appreciable variation in the $\sigma\left(V_{0}\right)-I$ relation. As the spatial resolution is decreased, however, the statistical sample (number of spectra) decreases, and sta-

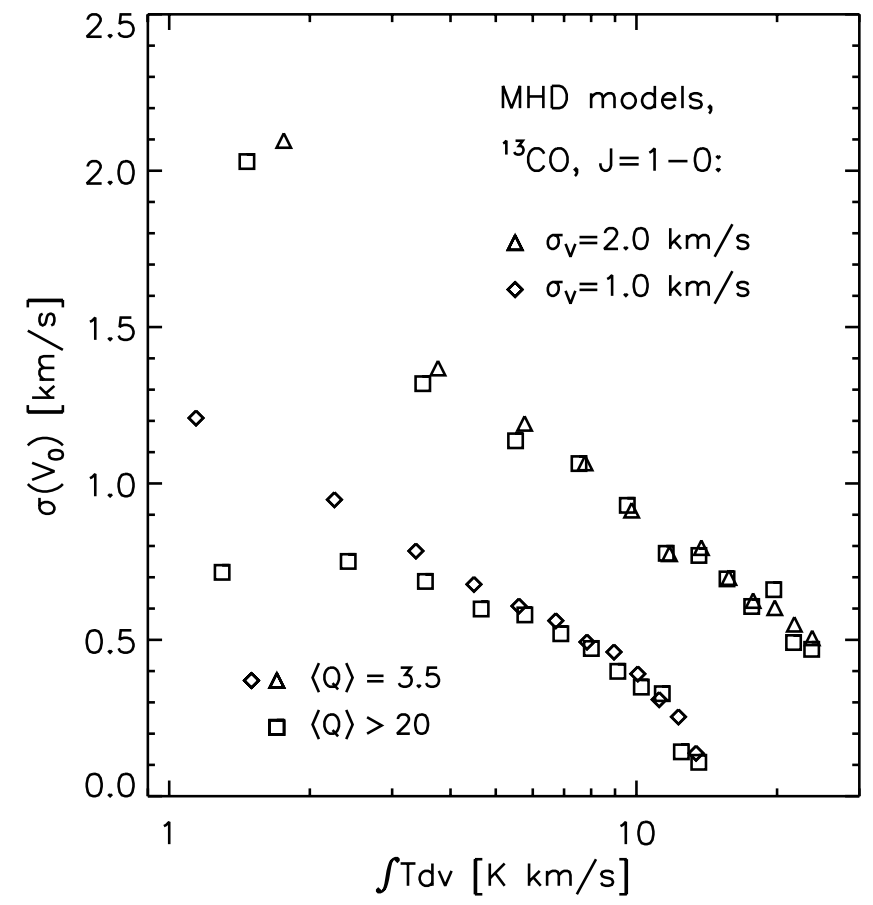

tistical fluctuations [deviations around the high-resolution $\sigma\left(V_{0}\right)-I$ relation] become progressively more important.

\section{OBSERVATIONAL $\sigma\left(V_{0}\right)-I$ RELATION}

We have shown in the previous section that the $\sigma\left(V_{0}\right)-I$ relation is sensitive to the value of the rms noise and to the spectral resolution. In the following plots, in which we compare different spectral maps from observations and models, we have therefore added noise to the spectra and increased the velocity channel width $d v$ to match the map with the largest noise and $d v$. We have not modified the spatial resolution in any map, since that has no systematic effect on the $\sigma\left(V_{0}\right)-I$ relation, as discussed above.

The left panel of Figure 6 shows the $\sigma\left(V_{0}\right)-I$ relation for the maps of MC complexes introduced in $\S 3$. For the Rosette MC complex we have used the portion of the full Blitz \& Stark (1986) map that matches the Heyer et al. (in preparation) map. The two models used for the comparison have $\sigma_{v}=2.0 \mathrm{~km} \mathrm{~s}^{-1}$, similar to Rosette and Perseus, and $\sigma_{v}=1.0 \mathrm{~km} \mathrm{~s}^{-1}$, similar to Taurus. It is remarkable that the Rosette and Perseus MC complexes have indistinguishable $\sigma\left(V_{0}\right)-I$ relations, which are also coincident with the theoretical prediction (Fig. 6, squares), for the same value of $\sigma_{v}$. The result for Taurus is also in good agreement with the $\sigma_{v}=1.0 \mathrm{~km} \mathrm{~s}^{-1}$ model. Horizontal shifts could be expected in the plot, since different MC complexes can have different surface density. However, MCs and MC complexes are known to approximately follow the Larson relation between density and size (eq. [2]), which implies roughly constant surface density and small horizontal shifts in the plot.

To compute the plots in the left panel of Figure 6, all maps have been treated to make their rms noise and veloc-

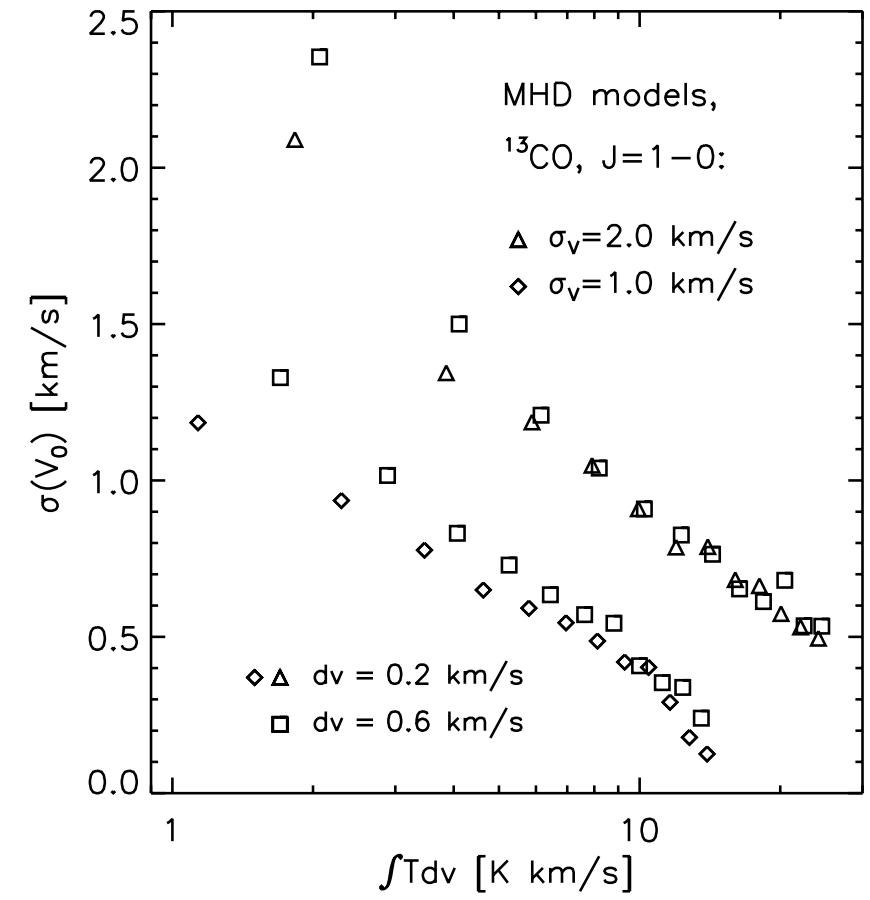

FIG. 5.-Effect of noise and spectral resolution. Left: Conditioned rms velocity centroid vs. intensity. Triangles and diamonds are as in Fig. 4, while squares have higher spectrum quality $\langle Q\rangle(\mathrm{S} / \mathrm{N})$. Different values of $\langle Q\rangle$ are obtained by adding different levels of noise to the synthetic spectra; $\langle Q\rangle=3.5$ is typical of the observational maps used in this work. The plot is not sensitive to the decreasing noise, for $\langle Q\rangle>20$. Right: Same as left, but squares are for lower spectral resolution (the velocity channels of the synthetic spectra are increased from $d v=0.2$ to $0.6 \mathrm{~km} \mathrm{~s}^{-1}$ ). Clearly, both the level of noise and the spectral resolution must be taken into account when comparing different maps. Integrated intensity values are binned over 12 linear intervals between the lowest and the highest values in the map, as in Fig. 4. [See the electronic edition of the Journal for a color version of this figure.] 

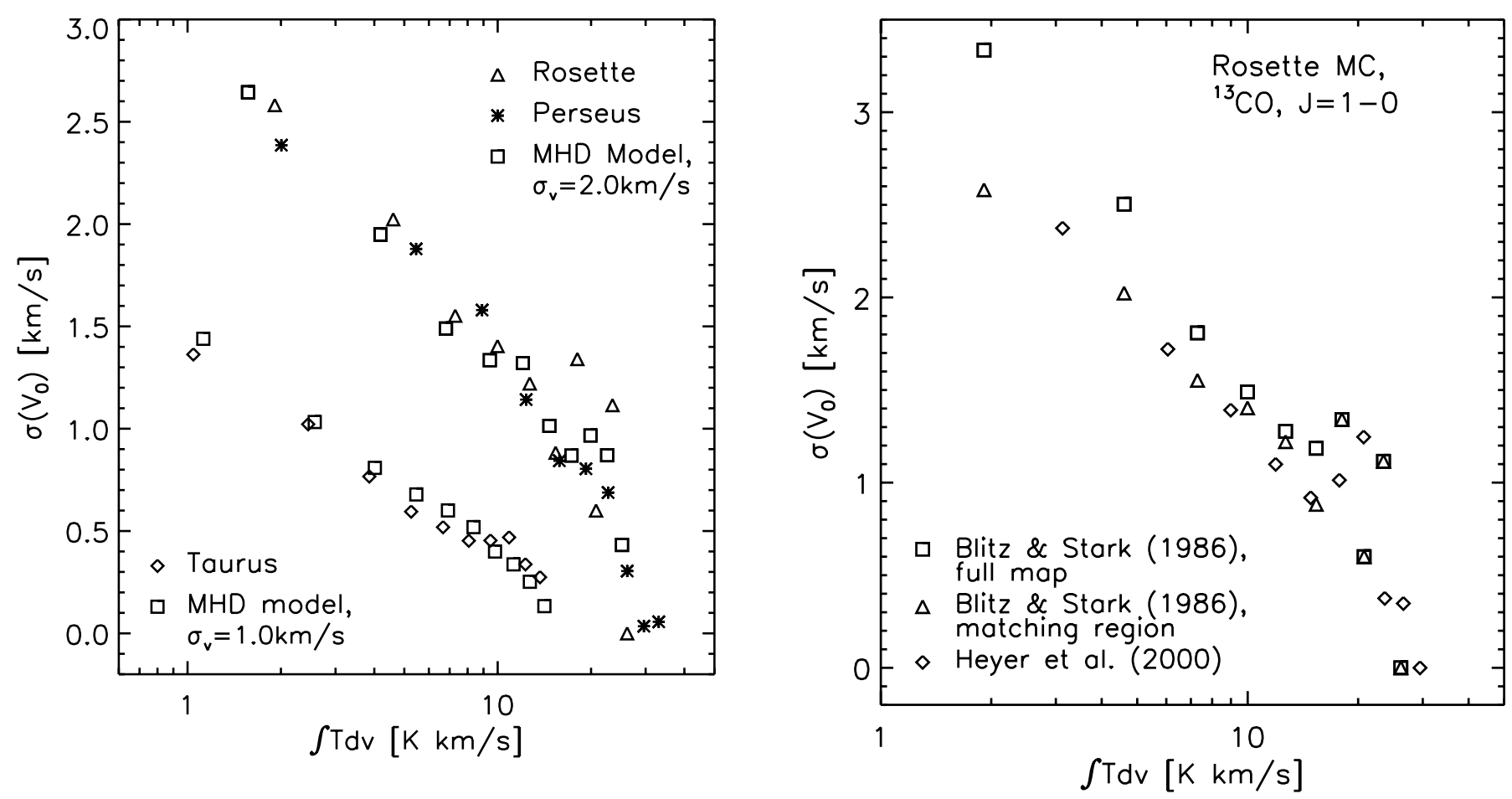

FIG. 6.-Observational $\sigma\left(V_{0}\right)-I$ relation. Left: Conditioned rms velocity centroid vs. intensity for different MC complexes: Rosette, Perseus, and Taurus. Squares are for maps of synthetic spectra with total rms velocity $\sigma_{v}=1.0$ and $2.0 \mathrm{~km} \mathrm{~s}^{-1}$. Right: Comparison of the two maps of the Rosette MC complex (see text for details). Integrated intensity values are binned over 10 linear intervals between the lowest and the highest values in the map. [See the electronic edition of the Journal for a color version of this figure.]

ity resolution equal. This is achieved by rebinning spectral profiles into a smaller number of velocity channels, and by adding noise when necessary. In order to check that the $\sigma\left(V_{0}\right)-I$ relations from different maps treated in this way are really comparable, we have computed the $\sigma\left(V_{0}\right)-I$ relation for the Rosette MC complex using both the Heyer et al. (in preparation) map and the portion of the Blitz \& Stark (1986) map that matches the region covered by the Heyer et al. map. The result is plotted in the right panel of Figure 6 . The velocity resolution of the Heyer et al. map has been decreased from $d v=0.06$ to $0.68 \mathrm{~km} \mathrm{~s}^{-1}$, and noise has been added, to exactly match the velocity resolution and rms noise in the Blitz \& Stark map. As can be seen in the right panel of Figure 6, after this drastic treatment of the higher resolution map, the $\sigma\left(V_{0}\right)-I$ relations for the two maps are practically indistinguishable from each other, which supports the validity of the comparison of different maps (Fig. 6, left; the case of the full Blitz \& Stark map is plotted in the right panel, by squares). The full map has a higher total rms velocity, $\sigma_{v}=2.4 \mathrm{~km} \mathrm{~s}^{-1}$, than the portion that matches the Heyer et al. map, and its $\sigma\left(V_{0}\right)-I$ relation is therefore steeper, as expected.

\section{DISCUSSION AND CONCLUSIONS}

The origin of proto-stellar cores is a fundamental problem in our understanding of the process of star formation. Models that have been proposed to describe protostellar cores are based on (1) static or quasi-static equilibrium (e.g., Curry \& McKee 2000; Fiege \& Pudritz 2000), (2) thermal instability (e.g., Yoshii \& Sabano 1980; Gilden 1984; Graziani \& Black 1987), (3) gravitational instability through ambipolar diffusion (e.g., Basu \& Mouschovias 1994; Nakamura, Hanawa, \& Nakano 1995; Indebetouw \& Zweibel 2000; Ciolek \& Basu 2000), (4) nonlinear Alfvén waves (e.g., Carlberg \& Pudritz 1990; Elmegreen 1990, 1997, 1999), (5) clump collisions (e.g., Gilden 1984; Kimura \& Tosa 1996), and (6) supersonic turbulence (e.g., Elmegreen 1993; Klessen, Burkert, \& Bate 1998; Klessen et al. 2000; Padoan et al. 2000a).

Many detailed comparisons between observational data and models, which support the idea of the turbulent origin of the structure and kinematics of molecular clouds, have been presented in our previous papers (Padoan et al. 1997, 1998, 1999, 2000a; Padoan \& Nordlund 1999). Models of numerical turbulence can be compared with the observations by computing (1) synthetic stellar extinction measurements, (2) synthetic spectral maps of molecular transitions, (3) synthetic Zeeman splitting measurements, and (4) synthetic polarization maps.

Maps of synthetic spectra contain a lot of information about the structure, kinematic, and thermal properties of molecular clouds, and can be analyzed with different statistical tools. In this work we have presented a new statistical method for analyzing spectral-line maps, which is very useful because it probes directly a very general property of supersonic turbulence, that is, the fact that dense gas traces a gas component with a smaller velocity dispersion than lower density gas. This property arises because the gas density is enhanced in regions where the large-scale turbulent flow converges (compressions) and the kinetic energy of the turbulent flow is dissipated by shocks. If local compressions are instead due to local instabilities (e.g., 
gravitational instability, or gravitational instability mediated by ambipolar diffusion), and the large-scale motions are only the consequence of local instabilities (as it should be in a self-consistent picture), gas density increases with the flow velocity dispersion (see, e.g., the model by Indebetouw \& Zweibel 2000), contrary to the observational evidence presented in this work.

The main conclusion of this work is that every model for the origin of molecular cloud structure and proto-stellar cores should be tested against the $\sigma\left(V_{0}\right)-I$ relation. Turbulent fragmentation provides a realistic scenario for the origin of proto-stellar cores, which satisfies this new observational constraint.

We are grateful to Edith Falgarone and Phil Myers for discussions that stimulated this work, and to the referee Jim Stone for a number of useful comments. This work was supported by NSF grant AST 97-21455. Åke Nordlund acknowledges partial support by the Danish National Research Foundation through its establishment of the Theoretical Astrophysics Center.
Ballesteros-Paredes, J., Hartmann, L., \& Vázquez-Semadeni, E. 1999, ApJ, 527,285

Basu, S., \& Mouschovias, T. C. 1994, ApJ, 432, 720

Bazell, D., \& Désert, F. X. 1988, ApJ, 333, 353

Beech, M. 1987, Ap\&SS, 133, 193

Blitz, L., \& Stark, A. A. 1986, ApJ, 300, L89

Carlberg, R. G., \& Pudritz, R. E. 1990, MNRAS, 247, 353

Ciolek, G. E., \& Basu, S. 2000, ApJ, 529, 925

Crutcher, R. M. 1999, ApJ, 520, 706

Curry, C. L., \& McKee, C. F. 2000, ApJ, 528, 734

Dame, T. M., Elmegreen, B. G., Cohen, R. S., \& Thaddeus, P. 1986, ApJ, 305, 892

Dickman, R. L., Margulis, M., \& Horvath, M. A. 1990, ApJ, 365, 586

Elmegreen, B. G. 1990, ApJ, 361, L77

. 1993, ApJ, 419, L29

.1997, ApJ, 480, 674
-1999, ApJ 527, 266

Elmegreen, B. G., \& Falgarone, E. 1996, ApJ, 471, 816

Falgarone, E., \& Pérault, M. 1987, in Physical Processes in Interstellar

Clouds, ed. G. Morfil \& M. Scholer (Dordrecht: Reidel), 59

Falgarone, E., \& Phillips, T. G. 1990, ApJ, 359, 344

Falgarone, E., Phillips, T. G., \& Walker, C. 1991, ApJ, 378, 186

Falgarone, E., Pineau des Forets, G., \& Roueff, E. 1995, A\&A, 300, 870

Falgarone, E., \& Puget, J. 1995, A\&A, 293, 840

Falgarone, E., Puget, J. L., \& Pérault, M. 1992, A\&A, 257, 715

Fiebig, D., \& Güsten, R. 1989, A\&A, 214, 333

Fiege, J. D., \& Pudritz, R. E. 2000, ApJ, 544, 830

Galsgaard, K., \& Nordlund, A. 1996, J. Geophys. Res., 101, 13445

Gammie, C. F., \& Ostriker, E. C. 1996, ApJ, 466, 814

Gilden, D. L. 1984, ApJ, 283, 679

Graziani, F. R., \& Black, D. C. 1987, Astrophys. Lett., 25, 235

Henriksen, R. N. 1991, ApJ, 377, 500

Hetem, A., J., \& Lepine, J. R. D. 1993, A\&A, 270, 451

Hobson, M. P. 1992, MNRAS, 256, 457

Indebetouw, R., \& Zweibel, E. G. 2000, ApJ, 532, 361

Juvela, M. 1997, A\&A, 322, 943 . 1998, A\&A, 329, 659

Kimura, T., \& Tosa, M. 1993, ApJ, 406, 512 1996, A\&A, 308, 979

Kleiner, S. C., \& Dickman, R. L. 1985, ApJ, 295, 466

.1987, ApJ, 312, 837

Klessen, R. S., Burkert, A., \& Bate, M. R. 1998, ApJ, 501, L205

Klessen, R. S., Heitsch, F., \& Mac Low, M. 2000, ApJ, 535, 887

Larson, R. B. 1981, MNRAS, 194, 809

Lee, S., Lele, S. K., \& Moin, P. 1991, Phys. Fluids A, 3, 657

Leorat, J., Passot, T., \& Pouquet, A. 1990, MNRAS, 243, 293

Leung, C. M., Kutner, M. L., \& Mead, K. N. 1982, ApJ, 262, 583

Lis, D. C., Pety, J., Philips, T. G., \& Falgarone, E. 1996, ApJ, 463, 623

Mac Low, M. M. 1999, ApJ, 524, 169

Mac Low, M. M., Smith, M. D., Klessen, R. S., Burkert, A., \& Smith, M.

1999, Interstellar Turbulence, ed. J. Franco \& A. Carraminana

(Cambridge: Cambridge Univ. Press), 256

Miesch, M. S., \& Bally, J. 1994, ApJ, 429, 645

\section{REFERENCES}

Miesch, M. S., \& Scalo, J. M. 1995, ApJ, 450, L27

Miesch, M. S., Scalo, J. M., \& Bally, J. 1999, ApJ, 524, 895

Mizuno, A., Onishi, T., Yonekura, Y., Nagahama, T., Ogawa, H., \& Fukui, Y. 1995, ApJ, 445, L161

Myers, P. C. 1983, ApJ, 270, 105

Myers, P. C., \& Goodman, A. A. 1988, ApJ, 326, L27

Nakamura, F., Hanawa, T., \& Nakano, T. 1995, ApJ, 444, 770

Nordlund, A., \& Galsgaard, K. 1997, A 3D MHD Code for Parallel Computers (Tech. rep.; Astronomical Observatory, Copenhagen University

Nordlund, A., \& Padoan, P. 1999, in Interstellar Turbulence, ed. J. Franco \& A. Carraminana (Cambridge: Cambridge Univ. Press), 218

Nordlund, A., Stein, R. F., \& Galsgaard, K. 1996, in Applied Parallel Computing, ed. J. Dongarra, K. Madsen, \& J. Wazniewsky (Heidelberg: Springer), 450

Ostriker,E. C., Gammie, C. F., \& Stone, J. M. 1999, ApJ, 513, 259

Ostriker, E. C., Stone, J. M., \& Gammie, C. F. 2001, ApJ, 546, 980

Padoan, P. 1995, MNRAS, 277, 377

Padoan, P., Bally, J., Billawala, Y., Juvela, M., \& Nordlund, Å. 1999, ApJ, 525,318

Padoan, P., Jones, B., \& Nordlund, Å. 1997, ApJ, 474, 730

Padoan, P., Juvela, M., Bally, J., \& Nordlund, A. 1998, ApJ, 504, 300

Padoan, P., \& Nordlund, A. 1997, preprint (astro-ph/9706176)

.1999, ApJ, 526, 279

Padoan, P., Nordlund, Å., Rögnvaldsson, Ö., \& Goodman, A. A. 2000a, ApJ, submitted

Padoan, P., Rosolowsky, E. W., Goodman, A. A. 2000b, ApJ, in press

Padoan, P., Zweibel, E., \& Nordlund, Å. 2000c, ApJ, 540, 332

Passot, T., \& Pouquet, A. 1987, J. Fluid Mech., 181, 441

Passot, T., Pouquet, A., \& Woodward, P. 1988, A\&A, 197, 228

Passot, T., \& Vázquez-Semadeni, E., A., P. 1995, ApJ, 455, 536

Porter, D. H., Pouquet, A., \& Woodward, P. R. 1992, Phys. Rev. Lett., 68, 3156

1994, Phys. Fluids, 6, 2133

Quiroga, R. J. 1983, Ap\&SS, 93, 37

Rosolowsky, E. W., Goodman, A. A., Wilner, D. J., \& Williams, J. P. 1999, ApJ, 524, 887

Sanders, D. B., Scoville, N. Z., \& Solomon, P. M. 1985, ApJ, 289, 373

Scalo, J. M. 1984, ApJ, 277, 556

. 1990, in Physical Processes in Fragmentation and Star Formation, ed. R. Capuzzo-Dolcetta, C. Chiosi, \& A. D. Fazio (Dordrecht: Kluwer), 151

Scalo, J. M., Vázquez-Semadeni, E., Chappell, D., \& Passot, T. 1998, ApJ, 504,835

Stone, J. M., Ostriker, E. C., \& Gammie, C. F. 1998, ApJ, 508, L99

Vázquez-Semadeni, E. 1994, ApJ, 423, 681

Vázquez-Semadeni, E., Passot, T., \& Pouquet, A. 1996, ApJ, 473, 881

Vogelaar, M. G. R., \& Wakker, B. P. 1994, A\&A, 291, 557

von Weizsäcker, C. F. 1951, ApJ, 114, 165

Yoshii, Y., \& Sabano, Y. 1980, PASJ, 32, 229

Zimmermann, T., Stutzki, J., \& Winnewisser, G. 1992, in Evolution of Interstellar Matter and Dynamics of Galaxies, ed. J. Palous, W. B. Burton, \& P. O. Lindblad (Cambridge: Cambridge Univ. Press), 254 